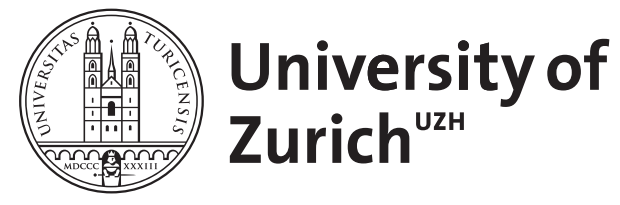

\title{
Das Gesundheitssystem in der Post-COVID-19-Epoche - Die Pandemiekrise und neue Wege des Pandemiemanagements
}

\author{
Kapitza, Thomas ; Greiff, Franz-Hubert ; Mann, Klaus
}

DOI: https://doi.org/10.32745/wcfm-6

Posted at the Zurich Open Repository and Archive, University of Zurich ZORA URL: https://doi.org/10.5167/uzh-199582

Scientific Publication in Electronic Form

Published Version

Originally published at:

Kapitza, Thomas; Greiff, Franz-Hubert; Mann, Klaus (2020). Das Gesundheitssystem in der PostCOVID-19-Epoche - Die Pandemiekrise und neue Wege des Pandemiemanagements. Berlin: Medizinisch Wissenschaftliche Verlagsgesellschaft.

DOI: https://doi.org/10.32745/wcfm-6 
Die in Deutschland aktuelle, derzeit medizinisch weitgehend beherrschte COVID-19-Pandemie muss zeitnah Anlass sein, das Pandemiemanagement weiterzuentwickeln und für zukünftige Bedrohungen krisenfest zu gestalten. Ein effizientes und transparentes Zusammenspiel von Wissenschaft, Gesundheitssystem, Wirtschaft, Politik und gesamtgesellschaftlichem Engagement sollte zum Wohle der Bürger*innen vorangetrieben werden.

Innerhalb einer ethischen Rahmensetzung sollte es zu einer Verbesserung und Bündelung wichtiger Maßnahmen kommen. Hierzu gehören eine verlässliche Daseinsvorsorge für die Bevölkerung, Erhalt der Vertrauenswürdigkeit in staatliche Institutionen und in die Solidargemeinschaft, Nachhaltigkeit, der Aufbau eines Nationalen Pandemierates, verantwortungsvoller Umgang mit Gesundheitsdaten, Ausbau eines digitalen Gesundheitssystems und Installation eines Frühwarnsystems sowie internationale Zusammenarbeit. In der jetzigen Post-COVID-19Epoche sollten Versorgungsstrategien weiterentwickelt werden, um insbesondere vulnerable Patienten- und Bevölkerungsgruppen zu schützen. Darüber sollten persönlich und wirtschaftlich gravierende Einschränkungen durch die staatlichen Institutionen angemessen und nachvollziehbar gehandhabt werden.

\section{Die aktuelle COVID-19-Pandemie als Lern- und Verbesserungschance}

Viele Gesundheitssysteme, politische Entscheider*Innen und ganze Gesellschaften müssen sich derzeit durch ihre Bürger*Innen daran messen lassen, wie erfolgreich sie die Herausforderungen der durch das SARS-CoV-2 Virus entstandenen Pandemie bewältigen ${ }^{1}$.

Ziel des Artikels ist es, aus einer übergeordneten Perspektive heraus das deutsche Gesundheitssystem für zukünftige, mögliche Virus-bedingte Bedrohungen der Bevölkerung krisenfester als bisher aufzustellen [1-10].

Innerhalb des anstehenden Entwicklungsprozesses sollte die ethische Rahmensetzung darin bestehen, dass die wichtigen gesundheitlichen und gesellschaftlichen Ziele, übergeordnet zu den Bestrebungen und Partikularzielen einzelner Interessengruppen im Gesundheitswesen, in den Vordergrund gestellt werden. Wichtigstes Ziel muss dabei bleiben, Krankheits- und Todesraten wirksam einzuschränken.

Der Erfolg zukünftiger Steuerungsmaßnahmen und politischer Entscheidungen wird in großem Umfang davon abhängen, ob es gelingen wird, das Vertrauen der Bevölkerung in die Leistungsfähigkeit des Gesundheitswesens und in die Entscheidungsfähigkeit und Umsetzungskompetenz der Politik zu erhalten und zu erweitern. Damit wird Vertrauen zu einer wichtigen Ressource gelingender Weiterentwicklung und ein vertrauensbasiertes Pandemiemanagement zu einer effizienten Option des Entwicklungsprozesses. Auch werden zukünftige digitale Präventions- und Versorgungsmöglichkeiten wie die Nationale Corona-App in ihrer Wirksamkeit hiervon abhängen $[11-13]$.

Die mit der ersten COVID-19-Infektionswelle in Deutschland verbundene Pandemiewahrnehmung [14, 15] hat sich tief in das Bewusstsein der Bevölkerung eingeprägt. Gegenwärtig scheint die Wiederherstellung eines ge-

${ }^{1}$ Zum Zeitpunkt des Redaktionsschluss dieses Beitrags (16.6.2020) galt für den Bereich der Bundesrepublik Deutschland die „erste PandemieInfektionswelle“ als überstanden. Eine weitere COVID-19-Infektionswelle war aus den vorliegenden epidemiologischen Daten heraus nicht erkennbar. Auch ist derzeit nicht vorherzusagen, ob und wann wirksame Therapien und/oder eine Schutzimpfung zur Verfügung stehen werden. 
sundheitlichen Sicherheitsgefühls in der Gesellschaft von ähnlich umfassender und den Lebensalltag durchdringender Bedeutung zu sein. Damit ist nicht nur das Gefühl einer Bedrohung der persönlichen Gesundheit im Familien-, Freundes- und Bekanntenkreis verbunden. Eine Gefährdung des bisher gewohnten materiell vergleichsweise gesicherten Lebensstils und die Angst vor persönlichen Wohlstands-, Freiheits- und LebensqualitätVerlusten wird ebenfalls empfunden.

Die aktuelle Verbesserung der COVID-19-Pandemie-Kennzahlen (Stand: Mitte Juni 2020) sollte nicht darüber hinwegtäuschen, dass die weiter bestehende Virus-Bedrohung in ihrer wellenförmig-phasenbezogenen Ausbreitung noch lange nicht vollständig in ihrem Schadenspotenzial wirksam eingegrenzt ist. Aus Vorsichtsgründen sollte deshalb worst case-bezogen davon ausgegangen werden, dass sich insbesondere Deutschland derzeit in einem Wellental zwischen der 1. Infektionswelle und einer möglichen neuen 2. Infektionswelle befindet. Es ist derzeit u.a. unklar, welche Amplitude dann die nächste(n) Infektionswelle(n) aufweisen werden, und wann wirksam schützende und nebenwirkungsarme Wirkstoffe für Arzneimittel und Impfstoffe verfügbar sein werden.

Das aktuelle Virus stellt wahrscheinlich nur eine von insgesamt ca. 20-40 Virusformen dar, welche auf die Menschheit kurzfristig übergreifen könnten [16, 17]. Das bedeutet, dass virale Gesundheitsbedrohungen zukünftig zu einem Dauerzustand werden könnte, welcher dann in einer sensibilisierten Gesellschaft zu bewältigen wäre.

Die Qualität eines deutschen Post-COVID-19-Gesundheitssystems bei der Pandemieabwehr und Pandemieprävention wird davon abhängen, ob optimierte Strukturen, Leistungsprozesse und Versorgungsergebnisse zukünftig dabei helfen werden, Krankheits- und Todesfälle zu minimieren, sowie die negativen Begleiteffekte wirtschaftlicher und gesellschaftlicher Art gering zu halten. Es ist davon auszugehen, dass die gegenwärtige Pandemiekrise den Gesundheitssektor langfristig beeinflussen wird.

In Pandemiezeiten sollte ein gutes Gesundheitssystem insbesondere die Eigenschaften „Schnelle Eingrenzung der Infektionen“ und „Wirksame Gegenmaßnahmen für die am stärksten vitalgefährdeten Bevölkerungsgruppen“ aufweisen. Wegen zukünftiger Strukturanpassungen (Intensivbetten-Aufstockungen; Medizingeräte usw.) ist damit zu rechnen, dass eine Wiederbelebung der gesundheitspolitischen, gesellschaftlichen und ethischen Diskussionen um angemessene Strukturvorhaltungsleistungen im Gesundheitssektor im Rahmen der staatlichen Daseinsvorsorge stattfinden wird.

Es sollte eine gesamtgesellschaftlich konsentierte Ausgangsposition werden, dass sich erfolgreiches Pandemiemanagement vor allem auf die Bevölkerungsbedürfnisse (u.a. Zugang zu hochwertiger Versorgung; Herstellung von Gesundheitssicherheit usw.) beziehen sollte. Dieses bedeutet nicht nur eine (wahrscheinlich unzureichende partielle) Optimierung spezifischer Versorgungsprozesse. Vielmehr ist auch die Notwendigkeit einer pandemieadäquaten zukünftigen Strukturgestaltung des Gesundheitssektors einschließlich (digital)technologischer Entwicklungsoptionen zu berücksichtigen. Eine Einführung neuer digitaler Versorgungstechnologien kann wichtige Lösungsoptionen erzeugen. Die Hoffnung allein auf einen umfassend problemlösenden Technologiefortschritt (Technologisches Narrativ) kann aber nicht der einzige Ansatz im Gesundheitssektor sein. Gesellschaftspolitische, wirtschaftspolitische und gesundheitspolitische Prioritäten und Gestaltungsansätze werden in Einklang zu bringen sein [18].

\section{Ethische Rahmensetzung für die Weiterentwicklung}

Um die Überlegungen zur zukünftigen Verbesserung der Pandemiefestigkeit des Gesundheitswesens zu unterstützen, ist neben dem durch das Grundgesetz festgelegten Werte-Kanon, eine allgemeine ethische Rahmensetzung als Orientierung für diesen Entwicklungsprozess hilfreich [19, 20].

Die nachfolgenden Werte-Überlegungen sollten dabei von Bedeutung sein:

\section{Gleichheit und Menschenwürde}

In Pandemiezeiten gilt als wichtiger gesamtgesellschaftlicher Konsens, dass alle Bürger*Innen den gleichen Anspruch auf Respektierung ihrer Menschenwürde haben. Die Vermeidung von Diskriminierungen in Pandemiezeiten, u.a. aufgrund von Lebensalter, Vorerkrankungen, weltanschaulichen Orientierungen, individuellen ökonomischen Möglichkeiten und daraus abgeleiteten Zugangserweiterungen/-einschränkungen zur medizinischen Versorgung, ist unverzichtbar [21][22, 23]. Gleiches gilt auch für die Diskriminierungsgefahr von Einzelpersonen aus der Verwendung bzw. Nicht-Verwendung nationaler COVID-19 Tracking Apps [24-26]. 


\section{Daseinsvorsorge für die gesundheitliche Sicherheit der Bürger*Innen}

Der Staat gewährleistet, in einer allgemeinen Analogie zum militärischen Sicherheitsbedürfnis, in der anstehenden Epoche permanenter Pandemie-Bedrohungen, die gesundheitliche Sicherheit seiner Bürger*Innen, und deckt damit ein gesellschaftliches Grundbedürfnis angemessen ab [27].

\section{Vertrauen und Vertrauenswürdigkeit}

Eine leidens- und schadensminimierende Pandemiebekämpfung [28] fördert den Erhalt und die Festigung des Vertrauens der Bevölkerung in den Staat. Die durch dieses Vertrauen gestärkte Leistungsfähigkeit der Gesellschaftspolitik und Sozialpolitik sichert die persönliche und gesellschaftliche Zukunft der Bürger*Innen. Die staatlichen Institutionen sind verpflichtet, das in sie gesetzte Vertrauen und den mit ihren Organisationen verbundenen Investitions- und Betriebsaufwand durch effektives Pandemiemanagement und gute Ergebnisse zu rechtfertigen.

\section{Freiheit und technologie- und datenethische Regelwerke}

Die Einhaltung der konstitutionellen Grundrechte im Kontext zukünftiger digitaler Technologien des Pandemiemanagements ist stets sicherzustellen und zu überprüfen. So darf beispielsweise die Verwendung von Tracking-Apps nicht die Freiheitsrechte und Autonomie der Anwender, z.B. durch eine Weiterverwendung generierter App-Daten für nicht-pandemiebezogene Nutzungen (u.a. für Überwachungszwecke; Social Profiling; privatwirtschaftliche Vertriebsprozesse usw.), einschränken [29-31]. Die Konzeption und Verwendung neuer Digitaltechnologien sollte nach Maßgabe technologieethischer und datenethischer Regelwerke [32] erfolgen.

\section{Mitgefühl und Solidarität}

Innergesellschaftlich sollten das persönliche Mitgefühl und die Solidarität (als gesellschaftliches Mitgefühl der Menschen untereinander) die Grundlage gemeinsamen Handelns in Krisenzeiten sein. Die unverzichtbare Notwendigkeit internationaler Zusammenarbeit sollte prägend wirken. Alle Beteiligten sollten bereit sein zu persönlichen Einschränkungen und ggfs. zu persönlichen Verzicht, wenn hierdurch die Pandemie wirksamer bekämpft werden kann. Alle pandemierelevanten Aktivitäten sollten sich am Ziel orientieren, die gesundheitliche Sicherheit der Bevölkerung in gesamtgesellschaftlicher Solidarität zu erhalten. Alle Aktivitäten müssen sich dabei im Einklang mit dem Leitbild einer freiheitlich-demokratisch organisierten Gesellschaftsordnung befinden.

Eine unsolidarische Aufspaltung der Bevölkerung in Nicht-Infizierte, Erkrankte und Gesundete (ggfs. als „Immune“ mit Immunitätsausweisen) und damit einhergehende Stigmatisierungsprozesse sind zu vermeiden. Damit kann ggfs. auch ein „Test-Paradoxon“ (Testung evtl. durch die Einzelperson unerwünscht, weil aufgrund der befürchteten Ergebnisse individuelle bzw. gruppenbezogene Nachteile entstehen könnten) vermieden werden.

\section{Gerechtigkeit}

Jeder hat das Recht und die Chance auf Versorgung, und keine Person mit ihren gesundheitlichen Problemen und Ängsten sollte durch die Gesellschaft zurückgelassen werden. Insbesondere gesundheitlich vulnerable Bevölkerungsgruppen sind durch das gemeinsame Handeln zu schützen und erfahren damit Wertschätzung. Das primär bedarfswirtschaftlich organisierte Gesundheitswesen in Deutschland verkörpert einen wichtigen Aspekt gesellschaftlich-sozialer Gerechtigkeit, weil es die diskriminierungsfreie Daseinsvorsorge durch den offenen Zugang zur Versorgung sicherstellt. Gerechtigkeit ist damit ein wichtiger Bestandteil der Werte-Struktur und der politischen, sozioökonomischen und technologischen Systemstruktur des Landes.

Vulnerable Bevölkerungs- und Patientengruppen (u.a. Risiko-Gruppen wie Diabetes Typ 2-Patient*innen, KHKund Schlaganfall-Patient*Innen, COPD-, Asthma-, onkologische Patient*Innen) stehen aufgrund ihrer pandemiebedingt wahrscheinlich höheren Vitalgefährdung unter besonderem Schutz.

\section{Reziprozität}

Zukünftiges Pandemiemanagement kann nur erfolgreich sein, wenn die Bürger*Innen nicht nur einen leistungsfähigen Staat erwarten, sondern gleichzeitig auch bereit sind, die staatlichen Entscheidungen umzusetzen. Dadurch helfen sie den staatlichen Institutionen, eine effiziente Pandemiebewältigung als gemeinsame Anstrengung aller Beteiligten zu erreichen. Von der Bevölkerung muss erwartet werden können, dass sie wissensund informationsbasierte hoheitliche Pandemiemaßnahmen unterstützt. 


\section{Selbstverantwortung des Staates}

Der freiheitlich-demokratisch organisierte Staat ist verantwortlich für die proaktive Planung und Umsetzung der notwendigen Veränderungen. Diese Planungen sollten stets verbunden sein mit dem Ziel der Sicherstellung eines leistungsfähigen Gesundheitssektors für die Allgemeinheit gerade in Zeiten aktueller und zukünftiger Pandemie-Bedrohungen. Eine Delegation dieser Verantwortung an nicht-staatliche Organisationen und einzelne Interessengruppen muss vermieden werden.

\section{Transparenz durch Konsensorientierung}

Die Diskussion des verbindlichen Entwicklungsprozesses hin zu einer „pandemiefesteren“ Gesellschaft ist mithilfe eines umfassend angelegten Diskurses [33, 34] unter dem Leitbild einer „Erklärenden Politik“ zu gestalten.

\section{Stabilität}

Um insbesondere die (massen-)psychologischen Effekte von Pandemien einzugrenzen und damit steuerbarer zu machen, ist gesellschaftliche Stabilität [35], erzeugt durch konsistentes Handeln der staatlichen Einrichtungen, ein wichtiger Wert. Es ist wichtig für die weitere Funktionsfähigkeit der Gesamtgesellschaft und damit auch für das Gesundheitssystem, dass eine Minderung persönlicher Ängste und die Vermittlung von Hoffnung auf Situationsverbesserung, als wichtige Handlungsziele des Pandemiemanagements berücksichtigt werden.

\section{Nachhaltigkeit}

Es ist dringend erforderlich, das grundsätzliche Zusammenleben zwischen Menschen und umgebender Natur (Mensch vs. Tierwelt/Pflanzenwelt) unter ökologischen Gesichtspunkten verstärkt zu berücksichtigen. Zoonosen (Überspringen von gefährlichen Viren aus der Tierwelt heraus auf menschliche Populationen) als Pandemieauslöser müssen verhindert werden. Gesundheitspolitik und Umweltpolitik sind deshalb eng miteinander verknüpft weiter zu entwickeln [36]. Zusätzlich ist auch die gesundheitsbeeinträchtigende Umweltverschmutzung (u.a. Wasserverunreinigungen; Schadstoffemissionen im Straßenverkehr) einzudämmen, um umweltbedingte Gesundheitsbeeinträchtigungen zu minimieren.

\section{Erste Vorschläge für Optimierungsansätze}

Die nachfolgenden Überlegungen sollten in die Strategie des Krisenmanagements im deutschen Gesundheitssystem einfließen:

\section{Themenfeld 1: Gesellschaft und Patienten}

\section{Gesundheitliche Daseinsvorsorge des Staates}

Ein vorsorgender Staat gestaltet proaktiv die gesundheitliche Daseinsvorsorge für die Cesellschaft und ihre Bürger. Der Staat sollte in diesem Zusammenhang die COVID-19-Pandemie u.a. als Chance zur Einleitung eines umfassenden Entwicklungsprozesses des Gemeinwesens nutzen. Die politisch Entscheidungsverantwortlichen berücksichtigen stets die freiheitlich-demokratische Gesellschaftsordnung und nutzen die demokratischen Prozesse der Willensbildung, Entscheidung [37] und Machtkontrolle. Eine proaktive Kommunikationspolitik der Bundesregierung und staatlichen Behörden sollte eine Stärkung des Vertrauens in die staatlichen Maßnahmen fördern.

\section{Zwang zur internationalen Zusammenarbeit}

Der existierende fachliche Zwang zur internationalen Zusammenarbeit im Rahmen der globalen Pandemiebekämpfung muss zukünftig dazu führen, dass eine effiziente und inhaltlich abgestimmte supranationale Pandemiepolitik ausgebaut wird (u.a. in den Handlungsfeldern Pandemieprävention/-detektion, Bekämpfungsprotokolle und Wirkungsevaluation [38], Pandemiemonitoring, Begleitschäden-Minderung). Der offensichtliche Zwang zu einer national und international abgestimmten Pandemiepolitik (u.a. bzgl. Analyse, Willensbildung, Entscheidung, Durchsetzung, Erfolgsmessung) mithilfe föderal legitimierter und steuernder Institutionen wird anerkannt. 


\section{Vermeidung von Zielkonflikten}

Gesundheitlicher Wohlstand muss sich in einer Balance mit ökonomischem Wohlstand befinden. Dabei sollte die gesundheitliche Lebensqualität der Bevölkerung als wichtiges Ziel im Vordergrund stehen, und volkswirtschaftliche Zielsetzungen diesem Ziel dienen. Zielkonflikte zwischen der Vermeidung volkswirtschaftlicher Pandemiefolgen [39] und Gesundheitsthemen sind in Pandemiezeiten zu benennen [40-44]. Gesundheitspolitik ist als Stabilitätspolitik und gleichzeitig als gesellschaftsweites „Angstmanagement“ aufzufassen. Pandemiebewältigung ist nicht ein ausschließlich rationaler Entwicklungsprozess.

Eine klare gesellschaftspolitische Prioritätensetzung ist notwendig bezüglich der Entscheidungen zur staatlichen Finanzierung der zukünftig erforderlichen Pandemie-Aufgaben [45, 46] (u.a. Vorhaltung von Versorgungskapazitäten, wissenschaftlicher Kompetenzaufbau, Ausbildung von Fachkräften).

Die Zielhierarchie der zukünftigen Pandemiepolitik sollte sein, weitgehend Krankheits- und Todesfälle zu vermeiden, sowie gleichzeitig die damit verbundenen Begleitschäden insbesondere wirtschaftlicher Art zu beherrschen $[47,48]$.

\section{Aufbau eines Nationalen Pandemierats}

Es wird ein Nationaler Pandemierat (NPR) aufgebaut, der aus einer multidisziplinären Perspektive heraus und unter der Kontrolle des Parlaments das multidisziplinäre Pandemiewissen zusammenführt. Der NPR bereitet alle Pandemiemanagement-Entscheidungen der politischen Entscheidungsträger durch datenbasierte rationale Vorschläge und unter Berücksichtigung der ethischen Vorgaben vor. Die Auswirkungen der getroffenen Entscheidungen werden wissenschaftlich evaluiert. Das Robert-Koch-Institut (RKI) sollte zukünftig die organisatorische Plattform für den NPR zur Verfügung stellen.

Es werden in Intervallen von ca. 1-2 Jahren regelmäßige Stresstests für die im Pandemiemanagement aktiven Task Force-Institutionen (NPR, RKI usw.) durch die zuständigen Fachaufsichtsbehörden durchgeführt. Dabei wird auch das aktuelle medizinisch-wissenschaftliche Fachwissen (u.a. Epidemiologie, Virologie, Hygieneforschung, Katastrophenmedizin) bilanziert und Empfehlungen für zukünftige Forschungsschwerpunkte gegeben.

\section{Verwendung von Gesundheitsdaten}

Daten (insbesondere persönliche Gesundheitsdaten und Versorgungsdaten der Bevölkerung) sind eine gesamtgesellschaftliche Ressource, deren Nutzung in Pandemiezeiten stets und zuerst gesamtgesellschaftlichen Zielen dienen sollte (u.a. Durchführung eines datengestützten Pandemiebekämpfung). Neue digitale Datenkonzepte der Öffentlichen Hand, insbesondere für den Gesundheitssektor und für Gesamteuropa, sollten unter multilateralen Aspekten des Pandemiemanagements ausgestaltet werden.

Alle Versorgungsleistungen sollten durch die Leistungserbringer so dokumentiert werden, dass sie als administrative Versorgungsdaten und klinische Patientendaten für die wissenschaftliche Verwertung nutzbar sind. Alle generierten Daten bleiben stets im Zugriff und in der hoheitlichen Verantwortung des Staates als „Datentreuhänder“ [49, 5o]. Sie können auf Antrag zu wissenschaftlichen Zwecken (dann verbunden mit einer umfassenden Publikationsverpflichtung zu den darauf beruhenden Forschungsergebnissen) in datenschutzrechtlich geeigneter Form an Dritte ausgehändigt werden.

\section{Umsetzung von Pandemiemaßnahmen}

In Pandemiezeiten sollte eine zeitlich begrenzte, vernunftbegründete und nach Pandemiebewältigung vollständig zurückgeführte Einschränkung bürgerlicher Freiheitsrechte möglich sein. Zu den einzelnen Maßnahmen wird parallel ein Ethikmonitoring durch eine neutrale Institution durchgeführt.

Die Bundesregierung und Bundesländer sind verpflichtet, den gemeinsamen Handlungsrahmen eines wirksamen Pandemiemanagements durch proaktive Selbstgestaltung unter Berücksichtigung ethischer Werte, klinischer Behandlungskapazitäten und der Personal- und Sachressourcen (u.a. Arzneimittel, Medizintechnische Gerätschaften [51], Medizinisch-pflegerische Fachkompetenz, Gesundheits- und Versorgungsdaten) festzulegen. Innerhalb des gesetzten Rahmens sind die jeweiligen Versorgungsakteure und Interessengruppen zum effizienten gemeinsamen Handeln zu verpflichten.

\section{Installation eines Pandemie-Frühwarnsystems}

Der staatliche Gesundheitsschutz der Bevölkerung sollte mithilfe von Investitionen in ein neues neutrales, nationales und internationales Frühwarnsystem für übertragbare Erkrankungen und eine kontinuierliche Prüfung der gesundheitlichen Bedrohungslage angestrebt werden. Um die Gesellschaft besser schützen zu können, ist eine Definition der gesellschaftlich systemrelevanten Pandemiebekämpfungsinstrumente und der hierfür not- 
wendigen und dauerhaft vorzuhaltenden Infrastruktur (insbesondere Technologie [52], Bauten, Ausstattung) notwendig.

\section{Epidemiologische Gesundheitsinformationen und Hysterieprävention}

Die Notwendigkeit klarer und allgemeinverständlicher „epidemiologischer Gesundheitsinformationen“ für die Bevölkerung und daraus abzuleitende Handlungsbegründungen wird anerkannt. Fachlich abgestimmte Botschaften aus dem NPR, aus den Expertenkreisen und aus regionalen bzw. lokalen Behörden stehen im Vordergrund. Primär nationale wissenschaftliche Datenquellen ermöglichen die Generierung der aktuellen Kennzahlen zur Pandemieentwicklung. Ein zeitnaher Abgleich der Anzahl der infizierten, sowie behandlungsbedürftig infizierten und behandlungsbedürftig Erkrankten mit den verfügbaren klinischen Versorgungskapazitäten ist sicherzustellen und zu kommunizieren [53].

Erfolgreiches Pandemiemanagement ist als gesundheitliche und als psychologisch-mentale Herausforderung zu sehen. Deshalb erfolgt zur Vermeidung von Falschnachrichten und Ähnlichem eine kontinuierliche und systematische Medien- und kommunikationswissenschaftliche Analyse der in den Medien jeweils aktuell verwendeten Themen-Narrative. Ziel ist die Erkennung und Gegensteuerung der Entstehung einer realitätsverfremdenden Wirkung dieser Falschnachrichten $[54,55]$. Alle Social-Media-Plattformbetreiber werden verpflichtet zur Sicherstellung einer redaktionellen Mindestqualität der Beiträge auf ihrer jeweiligen Plattform.

\section{Themenfeld 2: Gesundheitswesen}

Für den Gesundheitssektor werden die folgenden fachlichen Vorschläge in die Diskussionen eingebracht:

\section{Neuer Teilbereich des Gesundheitssektors}

Das Pandemiemanagement wird, neben den bereits bestehenden Schwerpunkten Prävention, Akutversorgung (Krisenversorgung einschließlich Intensivversorgung [56], Rehabilitation und Gesundheitswissen-Aufbau für persönliche Gesundheitskompetenz), zu einem neuen eigenständigen Teilbereich des Gesundheitssektors und der bestehenden gesundheitsrechtlichen bzw. sozialrechtlichen Gesamtstruktur. Als neuer zusätzlicher und eigenständiger Leistungsbereich innerhalb des Gesundheitswesens wird das Pandemiemanagement flächendeckend, übersektoral und kompetenzbündelnd organisiert.

Die sozialrechtliche Umsetzung der Vergütung für die pandemiebezogene Patientenversorgung erfolgt umfassend im Sozialgesetzbuch SGB V und wird damit Bestandteil des Leistungskatalogs der Gesetzlichen Krankenversicherung. Hierbei werden die bisherigen unbestimmten Rechtsbegriffe „ausreichend“, „zweckmäßig“ und „notwendig“ unter Einbezug des Deutschen Ethikrats zusätzlich ethisch-normativ im Pandemie-Kontext interpretiert und dadurch inhaltlich festgelegt. Die Vermeidung von Unter- bzw. Überversorgung wird durch die Kriterien und Vorgaben des aktualisierten Nationalen Pandemieplans unter Federführung des NPR erreicht.

Krankenhäuser sollten zukünftig in der Lage sein, zwei Patientenströme gleichzeitig zu versorgen: Nichtpandemiebedingt erkrankte Patient*Innen und pandemieassoziierte Erkrankungsfälle. Dieser neue Versorgungsalltag wird den Klinikbetrieb zukünftig beeinflussen [57-61]. Die bauliche und personelle Infrastruktur ist hierfür anzupassen $[62,63]$.

Das nationale Finanzierungssystem (insbesondere Leistungsentgelte und Investitionsmittel) wird für den Gesundheitssektor und die Leistungserbringer weiterentwickelt. Vor dem Hintergrund der föderalistischen Organisation des Gemeinwesens werden die Gebietskörperschaften (Bund, Bundesland, Landkreise, Städte und Gemeinden) durch eine quotale Leistungsfinanzierung, anteilige Vorhaltungsfinanzierung und durch die anteilige Finanzierung von versorgungswissenschaftlichem Wissensaufbau und von Pandemieanalyse-Technologien angemessen belastet.

\section{Nationale S3-Leitlinie „Pandemiemanagement“}

Die medizinische Indikationsstellung erfolgt durch pandemiekompetente Ärztinnen und Ärzte auf Basis definierter klinischer Versorgungsprozesse und einer für Forschungszwecke verwendbaren Behandlungs- und Ergebnisdokumentation. Die Basis hierfür sollte eine neuartige Nationale S3-Leitlinie: Pandemiemanagement sein. Die sozialrechtliche Leistungsvergütung für Pandemiepatienten als anteilige Finanzierung der Versorgungseinrichtungen folgt dabei der dokumentierten Indikationsstellung.

\section{Ausbau des digitalen Gesundheitssystems}

Es erfolgt der Ausbau des digitalen Gesundheitssystems unter Anwendung neuer Technologien (u.a. AI bzw. Machine Learning, Deep Learning). Diese Anwendungen haben das vorrangige Ziel, eine schnellere infektionsbe- 
zogene Mustererkennung und bessere Reaktionsmöglichkeiten im gesundheitlichen Krisenfall zu ermöglichen. Die Nutzung digitaler Technologien auf Basis valider Gesundheitsdaten (u.a. auf Basis von dezentraler Datengenerierung, Mustererkennung und zentral koordinierter Auswertung) soll die Wirksamkeitsevaluation der durchgeführten Versorgungsmaßnahmen beschleunigen. Das gesundheitsökonomische Effizienzmonitoring für Pandemiemaßnahmen (u.a. Public Health-Messkonzepte, RCT Randomized Controlled Trials, Real World Evidence RWE-Tools für pharmazeutische Wirkstoffe) wird verbindlich installiert. Der pandemiepräventive Einsatz digitaler Technologien wird sich zukünftig verstetigen [64]. Es bleibt abzuwarten, inwiefern die nationale „CoronaAPP“ die mit ihr zusammenhängenden Erwartungen erfüllen kann, und ob sie auf eine hohe Akzeptanz bei allen Bürger*innen treffen wird.

\section{Entitäten-spezifische Versorgungsstrategien}

Es werden zukunftsorientierte Entitäten-spezifische Versorgungsstrategien z.B. für vulnerable Patientengruppen und Bevölkerungsgruppen für zukünftige Pandemiezeiten erarbeitet (z.B. Chroniker, versorgungsdringliche Patient*Innen wie z.B. onkologisch versorgungsbedürftige Personen, ältere Menschen, Menschen mit Behinderungen, sozioökonomisch risikobelastete Menschen usw.).

\section{Ausblick}

Um ein leistungsfähiges zukünftiges Pandemiemanagement im Kontext eines freiheitlich-demokratischen und öffentlich finanzierten Gesundheitssystems aufzubauen, sind inhaltlich-fachliche und ethische Zielsetzungen zu berücksichtigen.

Das zukünftige Pandemiemanagement sollte die pandemierelevanten und miteinander vielfältig verknüpften Themen der Bereiche bzw. Handlungsebenen Cesamtgesellschaft, Bevölkerung, Patient*Innen und das Gesundheitswesen mit seinen Leistungserbringern und seinen Versorgungsleistungen umfassen.

Die Post-COVID-19-Epoche bietet die zeitliche Chance für die notwendigen Veränderungen, welche Menschenleben retten und Leiden vermindern könnten.

\section{Literatur}

1. LEOPOLDINA. Dritte Ad-hoc-Stellungnahme: Coronavirus-Pandemie - Die Krise nachhaltig über-winden (13.04.2020). Im Internet https://www.leopoldina.org/publikationen/detailansicht/publication/leopoldina-stellungnahmen-zur-coronavirus-pandemie-2020/; Stand: 14.04.2020

2. LEOPOLDINA. Stellungnahme 4 zur Coronavirus-Pandemie: Medizinische Versorgung und patientennahe Forschung in einem adaptiven Gesundheitssystem (27.05.2020). Im Internet: https://www.leopoldina.org/publikationen/detailansicht/publication/leopoldinastellungnahmen-zur-coronavirus-pandemie-2020/; Stand: 28.05.2020

3. Robert Koch-Institut. Nationaler Pandemieplan Teil I - Strukturen und Maßnahmen. RKI-Bib1 (Robert Koch-Institut); 2017. doi:10.17886/rkipubl-2017-005

4. Robert Koch-Institut. Nationaler Pandemieplan Teil II. RKI-Bib1 (Robert Koch-Institut); 2017. doi:10.17886/rkipubl-2016-004.5

5. Romanes M, Kurth F. Gemeinsam die Coronavirus-Pandemie eindämmen - Evaluation der Chancen und Risiken von Contact-Tracing-Apps Nutzungsrecht: Verein Ethik und Medizin Schweiz VEMS, Verwendung der Texte, auch (Mai 2020); Stand: 08.06.2020

6. Whiting K. How the world can 'reset' itself after COVID-19 - according to these experts (03.06.2020). Im Internet: https://intelligence.weforum.org/topics/a1G0X00000606EHUA0?tab=publications; Stand: 05.06.2020

7. Abele-Brehm A, Dreier H, Fuest C, Grimm V, Kräusslich H-G, Krause G, Leonhard M, Lohse A, Lohse M, Mansky T, Peichl A, Schmid R, Wess G, Woopen C. Die Bekämpfung der Coronavirus-Pandemie tragfähig gestalten. Empfehlungen für eine flexible, risikoadaptierte Strategie (02.04.2020). Im Internet: https://www.ifo.de/publikationen/2020/monographie-autorenschaft/die-bekaempfung-der-coronavirus-pandemietragfaehig; Stand: 05.05 .2020

8. Ärztekammer Westfalen-Lippe. Forderungskatalog des Vorstandes der Ärztekammer Westfalen-Lippe zur Stärkung des Gesundheitswesens in Zeiten von Infektionskrankheiten (05.06.2020). Im Internet:

https://www.aekwl.de/fileadmin/user_upload/aekwl/Presse/Pressemitteilungen/21_20_Corona_Forderungskatalog.pdf; Stand: 07.06.2020

9. o.V. Looking Back At COVID-19 From 2030 (13.05.2020). Im Internet: https://medicalfuturist.com/looking-back-at-covid-19-from2030/?utm_source=The\%20Medi-cal\%20Futurist\%20Newsletter\&utm_campaign=0cbaeac5a5EMAIL_CAMPAIGN_2020_05_19_Newsletter\&utm_medium=email\&utm_term=0_efd6a3cd08-0cbaeac5a5-420770529; Stand: 19.05 .2020

10. Cutler DM, Nikpay S, Huckman RS. The Business of Medicine in the Era of COVID-19. JAMA 2020: E1-E2. doi:10.1001/jama.2020.7242

11. Hinch R, Probert W, Nurtay Aea. Effective Configurations of a Digital Contact Tracing App: A report to NHSX (05.05.2020). Im Internet: https://cdn.theconversation.com/static_files/files/1009/Report__Effective_App_Configurations.pdf?1587531217; Stand: 12.06 .2020

12. Verbraucherzentrale Nordrhein-Westfalen und Verbraucherzentrale Rheinland-Pfalz. Corona-Warnung per App: Fragen und Antworten zur neuen Tracing-App (15.06.2020). Im Internet: https://www.verbraucherzentrale.de/wissen/digitale-welt/apps-und-software/coronawarnung per-app-fragen-und-antworten-zur-neuen-tracingapp-47466; Stand: 15.06 .2020

13. Vokinger KN, Nittas V, Witt CM, et al. Digital health and the COVID-19 epidemic: an assessment framework for apps from an epidemiological and legal perspective. Swiss Med Wkly 2020; 150: w20282. doi:10.4414/smw.2020.20282

14. Priddat B. Das Virus besiegen, heißt mit inm zu leben (19.05.2020). Im Internet: https://www.xing.com/news/insiders/articles/das-virusbesiegen-heisst-mit-ihm-zu-leben-3207017, Stand: 20.05.2020

15. o.V. Ärzte dringen auf weitere Vorkehrungen für Gesundheitskrisen. DÄB online, 15.06.2020. Im Internet: https://www.aerzteblatt.de/treffer?mode=s\&w0=17\&typ=1\&nid=113779\&s=Gesundheitskrisen; Stand: 16.06 .2020 
16. Gates B. Pandemic I: The First Modern Pandemic. The scientific advances we need to stop COVID-19 (23.04.2020). Im Internet: https://www.gatesnotes.com/Health/Pandemic-Innovation; Stand: 08.06.2020

17. Müller G. Gefährliche Zoonose-Erreger: „40 weitere Viren mit einem Pandemie-Potential wie Sars-CoV-2“ (03.05.2020). Im Internet: https://www.spiegel.de/wissenschaft/medizin/coronavirus-und-andere-zoonoseerreger-gerd-mueller-bundes-entwicklungsminister-warnt-vorden-gefahren-a-e4e42281-4ad1-460f-9ad3-818117be37b6; Stand: 04.05.2020

18. Fuchs VR. Health Care Policy After the COVID-19 Pandemic. JAMA 2020: E1-E2. doi:10.1001/jama.2020.10777

19. Deutscher Ethikrat. Solidarität und Verantwortung in der Corona-Krise. AD-HOC-EMPFEHLUNG (27.03.2020). Im Internet: https://www.ethikrat.org/fileadmin/Publikationen/Ad-hoc-Empfehlungen/deutsch/ad-hoc-empfehlung-corona-krise.pdf; Stand: 12.06 .2020

20. Joebges S, Biller-Andorno N. Ethics guidelines on COVID-19 triage-an emerging international consensus. Crit Care 2020; 24: 201. doi:10.1186/s13054-020-02927-1

21. Berwick DM. Choices for the "New Normal". JAMA 2020. doi:10.1001/jama.2020.6949

22. Schloeman |. Interview: „Der Ausdruck soziale Distanz ist ein Unding“. Der Philosoph Julian Nida-Rümelin über die Verantwortung des Einzelnen in der Corona-Krise, den Schutz von Risikogruppen und die zwei Wege, die er sieht, um Gesundheitssystem und Wirtschaft intakt zu halten. Süddeutsche Zeitung online, 22.05.2020. Im Internet: https://www.sueddeutsche.de/kultur/coronavirus-lockerungen-risikogruppenjulian-nida-ruemelin-interview-philosophie-1.4914827; Stand: 25.05.2020

23. Mann K, Kapitza T, Likar R, et al. 3-Länder-Manifest: Zeitenwende in der Medizin - Patienten-versorgung auf dem gefährlichen Weg in die Ökonomisierung und Industrialisierung. Deutsche Medizinische Wochenschrift 2019; 144: e145-e152. doi:10.1055/a-1024-8308

24. Cohen IG, Gostin LO, Weitzner D). Digital Smartphone Tracking for COVID-19: Public Health and Civil Liberties in Tension. JAMA 2020. doi:10.1001/jama.2020.8570

25. Hall MA, Studdert DM. Privileges and Immunity Certification During the COVID-19 Pandemic. JAMA 2020. doi:10.1001/jama.2020.7712

26. Persad G, Emanuel E). The Ethics of COVID-19 Immunity-Based Licenses ("Immunity Passports"). JAMA 2020. doi:10.1001/jama.2020.8102

27. Lehmann T. Städtetagspräsident Jung: „Auch im härtesten Epidemiefall muss die Daseinsvorsorge der Bürger gesichert sein“. Städtetagspräsident Jung fordert in der Krise finanzielle Unterstützung für die Kommunen - und macht sich dafür stark, den Menschen bald „wieder mehr Ausgang" zu ermöglichen. Spiegel Online, 30.03.2020. Im Internet: https://www.spiegel.de/politik/deutschland/burkhard-jung-auch-imhaertestenepidemiefall-; Stand: 07.06.2020

28. Shah SK, Miller FG, Darton TC, et al. Ethics of controlled human infection to address COVID-19. Science 2020; 368: 832-834 doi:10.1126/science.abc1076

29. DPA. Verfassungsrechtler Papier mahnt zu „Schutz der Freiheit“. Süddeutsche Zeitung online, 01.05.2020. Im Internet: www.sz.de/dpa.urnnewsml-dpa-com-20090101-200501-99-905221; Stand: 01.05.2020

30. Lovett L. Experts warn of technical limitations of contact tracing for coronavirus. This report comes as news breaks that the UK has decided not to use Apple and Google's contact-tracing tech as originally planned (27.04.2020). Im Internet: https://www.mobihealthnews.com/news/experts-warn-technical-limitations-contact-tracing-coronavirus; Stand: 02.05.2020

31. Shachar C, Engel J, Elwyn G. Implications for Telehealth in a Postpandemic Future: Regulatory and Privacy Issues. JAMA 2020 : E1-E2. doi:10.1001/jama.2020.7943

32. Tamo-Larrieux A. DSI Insights: Privatsphärenschutz während einer Pandemie (Mai 2020). Im Internet: https://www.inside-it.ch/de/post/dsiinsights-privatsphaerenschutz-waehrend-einer-pandemie-20200519; Stand: 27.05.2020

33. Bernstein J, Hutler B, Rider T, Faden R, Han H, Barnhill A. Grappling with the Ethics of Reopening: A Framework for Evaluating Reopening Policies (03.05.2020). Im Internet: https://bioethics.jhu.edu/research-and-outreach/covid-19-bioethics-expert-insights/resources-foraddressing-key-ethical-areas/grappling-with-the-ethics-of-social-distancing/; Stand: 05.05.2020

34. Fritz Z, Huxtable R, Ives I, et al. Ethical road map through the covid-19 pandemic. BMI 2020; 369: m2033. doi:10.1136/bmj.m2033

35. Lanzerath D. Die innere Krise durch Corona. F\&W 2020: 403-405

36. McCartney M. Medicine: before COVID-19, and after. The Lancet 2020; 395: 1248-1249. doi:10.1016/S0140-6736(20)30756-X

37. Bundesgesetzblatt. Gesetz zum Ausgleich COVID-19 bedingter finanzieller Belastungen der Krankenhäuser und weiterer Gesundheitseinrichtungen (COVID-19-Krankenhausentlastungsgesetz). COVID-19-Krankenhausentlastungsgesetz vom 27.03.2020, 580-586

38. Bendix A. Lockdowns worked, new research finds. Scientists estimate coronavirus measures stopped at least 60 million infections in the US and 3 million deaths in Europe. (09.06.2020). Im Internet: https://www.businessinsider.com/us-coronavirus-lockdowns-compared-tochinaeu-rope-; Stand: 10.06.2020

39. 0.V. Corona: Gesundheitswirtschaft weiterhin stark betroffen. Sonderauswertung der 3. DIHK-Blitzumfrage (28.05.2020). Im Internet: https://www.dihk.de/de/aktuelles-und-presse/presseinformationen/coronavirus-hat-auch-auf-die-gesundheitswirtschaft-negativeauswirkungen-24344; Stand: 14.06 .2020

40. Chen Y. The economic argument for saving lives (04.06.2020). Im Internet: https://www.kevinmd.com/blog/2020/06/the-economicargument-for-saving-lives.html; Stand: 14.06 .2020

41. Symons X. Ethicists double down on utilitarian framework for pandemics (31.05.2020). Im Internet: https://www.bioedge.org/bioethics/ethicists-double-down-on-utilitarian-framework-for-pandemics/13447; Stand: 02.06.2020

42. Romanes M, Warmuth W, Kurth F. Covid19 und Utilitarismus - Versuch einer medizinethischen Klärung (16.04.2020). Im Internet: http://docfind.ch/VEMSCovid19undUtilitarismus.pdf; Stand: 08.06.2020

43. Herles B. Wertedebatte: Warum Gesundheit und Wirtschaft zusammengehören (06.05.2020). Im Internet: https://www.capital.de/wirtschaftpolitik/wertedebatte-warum-gesundheit-und-wirtschaft-zusammengehoeren; Stand: 07.05.2020

44. Bello W. The Race to Replace a Dying Neoliberalism (19.05.2020). Im Internet: https://www.counterpunch.org/2020/05/19/the-race-toreplace-a-dying-neoliberalism; Stand: 20.05.2020

45. Beneker C. Konjunkturpaket: 10 Milliarden Euro für Kliniken, ÖGD und die „nationale Reserve“. Das plant die Regierung (10.06.2020). Im Internet:

https://deutsch.medscape.com/artikelansicht/4908975?src=mkm_demkt_200611_mscmrk_decoronavirus_nl\&uac=360512EY\&impID=241479 9\&faf=1\#vp_1; Stand: 14.06 .2020

46. o.V. Corona-Folgen bekämpfen, Wohlstand sichern, Zukunftsfähigkeit stärken. Ergebnis Koalitionsausschuss 3. Juni 2020, „Eckpunktepapier“ (03.06.2020). Im Internet:

https://www.bundesfinanzministerium.de/Content/DE/Standardartikel/Themen/Schlaglichter/Konjunkturpaket/2020-06-03 eckpunktepapier.pdf; Stand: 12.06 .2020

47. Kohn S. KM4 Analyse des Krisenmanagements (Kurzfassung). Interne Analyse des Corona-Krisenmanagements im Bundesministerium des Innern (Nicht autorisiertes Fachpapier der Abteilung „Krisenmanagement und Bevölkerungsschutz“, 05.05.2020: 91 Seiten

48. Externe Experten des Corona-Papiers BMI. Gemeinsame Pressemitteilung der externen Experten des Corona-Papiers aus dem Bundesministerium des Innern (11.05.2020); Stand: 12.05.2020

49. IQWIG. Konzept für ein nationales Gesundheitsportal (31.08.2018). Im Internet: https://www.iqwig.de/de/projekteergebnisse/projekte/gesundheitsinformation/p17-02-konzept-fuer-ein-nationales-gesundheitsportal.7849.html; Stand: 14.06 .2020 
50. Braunmühl P von, Klein M. Forschen mit Gesundheitsdaten: Warum ein Datentreuhänder so wichtig ist (28.04.2020). Im Internet: https://www.egovernment-computing.de/warum-ein-datentreuhaender-so-wichtig-ist-a-927892/; Stand: 02.05.2020

51. Savulescu I, Persson I, Wilkinson D. Utilitarianism and the pandemic. Bioethics 2020; 34: 620-632. doi:10.1111/bioe.12771

52. Sittig DF, Singh H. COVID-19 and the Need for a National Health Information Technology Infra-structure [Published online]. JAMA 2020: E1-E2. doi:10.1001/jama.2020.7239

53. Kolbeck C. Passen die Corona-Kapazitäten auf den Intensivstationen? (08.05.2020). Im Internet: https://www.medical-tribune.de/meinungund-dialog/artikel/passen-die-corona-kapazitaeten-auf-den-intensivstationen/; Stand: 14.06.2020

54. Rühle A. Die Allianz des Unsinns. Süddeutsche Zeitung online, 04.05.2020. Im Internet: https://www.sueddeutsche.de/kultur/hygiene-demos coronavirus-verschwoerungstheorien-1.4896453; Stand: 05.05.2020

55. 0.V. EPI-WIN - Health sector. Im Internet: https://www.who.int/teams/risk-communication/epi-win-updates; Stand: 14.06 .2020

56. DIVI Deutsche Interdisziplinäre Vereinigung für Intensiv- und Notfallmedizin. Entscheidungen über die Zuteilung von Ressourcen in der Notfall- und der Intensivmedizin im Kontext der COVID-19-Pandemie. Klinisch-ethische Empfehlungen der Deutschen Interdisziplinären Vereinigung für Intensiv- und Notfallmedizin (DIVI), der Deutschen Gesellschaft für Interdisziplinäre Notfall- und Akutmedizin (DGINA), der Deutschen Gesellschaft für Anästhesiologie und Intensivmedizin (DGAI) der Deutsche Gesellschaft für Internistische Intensivmedizin und Notfallmedizin (DGIIN) der Deutsche Gesellschaft für Pneumologie und Beatmungsmedizin (DGP) der Deutschen Gesellschaft für Palliativmedizin (DGP) und der Akademie für Ethik in der Medizin (AEM) Von den Fachgesellschaften verabschiedete Fassung vom 25.03.2020 (25.03.2020). Im Internet: https://www.divi.de/aktuelle-meldungen-intensivmedizin/covid-19-klinisch-ethische-empfehlungen-zur-entscheidung-ueber-diezuteilung-von-ressourcen-veroeffentlicht; Stand: 05.06.2020

57. Bundesministerium für Gesundheit. Ein neuer Alltag auch für den Klinikbetrieb in Deutschland (27.04.2020). Im Internet: https://www.bundesgesundheitsministerium.de/fileadmin/Dateien/3_Downloads/C/Coronavirus/Faktenpapier_Neuer_Klinikalltag.pdf Stand: 13.07 .2020

58. Baum G. Postpandemische Perspektiven. das Krankenhaus 2020; 112: 385

59. 0.V. Der Blick auf die Kliniken wird ein anderer sein. Interview mit Dr. Gerald Gaß, Präsident der Deutschen Krankenhausgesellschaft e.V (DKG)

60. Telgheder M. Krankenhauskonzern Helios - Wie der neue Alltag bei Europas größtem Klinikkonzern aussieht (02.06.2020). Im Internet: https://www.handelsblatt.com/technik/medizin/krankenhauskonzern-helios-wie-der-neue-alltag-bei-europas-groesstem-klinikkonzernaussieht/25865530.html?ticket=ST-452039-ozaefliLF09QkEbcRpzD-ap2; Stand: 05.06.2020

61. o.V. BMG-Stellungnahme: Ein neuer Alltag auch für den Klinikbetrieb in Deutschland (27.04.2020). Im Internet: https://www.bundesgesundheitsministerium.de/fileadmin/Dateien/3_Downloads/C/Coronavirus/Faktenpapier_Neuer_Klinikalltag.pdf Stand: 15.06 .2020

62. Chen S, Zhang Z, Yang I, et al. Fangcang shelter hospitals: a novel concept for responding to public health emergencies. The Lancet 2020; 395 : 1305-1314. doi:10.1016/S0140-6736(20)30744-3

63. Peterson A, Largent EA, Karlawish J. Ethics of reallocating ventilators in the covid-19 pandemic. BMI 2020; 369: m1828. doi:10.1136/bmj.m1828

64. Kobielus I. Schöne, neue Post-Corona-Welt (29.05.2020). Im Internet: https://www.cio.de/a/schoene-neue-post-coronawelt,3549081?tap=efe6a400176576509736e688cc77dce6\&utm_source=Healthcare\%20lT\&utm_medium=email\&utm_campaign=newsletter\&p m_cat[1]=mobile\%20os\&pm_cat[2]=mobi-le\%20client\&pm_cat[3]=mobilfunk\&pm_cat[4]=apps\&pm_cat[5]=mobile\%20plattformen\&r=5717 7511225852138\&lid=1512238\&pm_In=20; Stand: 16.06 .2020

\section{Thomas Kapitza}

Ö.b.u.v. Sachverständiger (IHK München und Oberbayern), Sachverständigenbüro Kapitza; Research Fellow IBME Institut für Biomedizinische Ethik und Medizingeschichte der Universität Zürich.

Diplom-Kaufmann (Univ.) Thomas Kapitza ist langjähriger national und international tätiger Gutachter für betriebswirtschaftliche Themen im Gesundheitswesen. Zu Fachthemen an der Schnittstelle Gesundheitsökonomie/Biomedizinische Ethik forscht er derzeit nebenberuflich am Institut für Biomedizinische Ethik der Universität Zürich. Zusätzlich ist er ökonomischer Experte für Digital Health und Mitautor des deutschen Klinik Codex/Ärzte Codex (2019).

Franz-Hubert Greiff

Beratender Arzt im Gesundheitsmanagement \& Qualitätsmanagement.

Arzt für Qualitätsmanagement Franz-Hubert Greiff ist langjährig im Krankenhausmanagement in diversen Kliniken tätig. Zu aktuellen Themen im Gesundheitsmanagement ist er seit 2015 mit dem Beratungsunternehmen medemus.de im deutschsprachigen Raum aktiv.

Prof. Dr. med. Klaus Mann

Em. Direktor der Klinik für Endokrinologie, Diabetologie und Stoffwechsel, Zentrallabor-Bereich Forschung \& Lehre Universitätsklinikum Essen.

Professor Mann ist Facharzt für Innere Medizin, Endokrinologe, Diabetologe und Androloge und ist derzeit tätig am Schilddrüsenzentrum Tegernsee. Er war langjähriger Sprecher der Sektion Schilddrüse der Deutschen Gesellschaft für Endokrinologie. Er ist Mitglied der Arbeitsgruppe Struktur - Krankenversorgung der Deutschen Gesellschaft für Innere Medizin DGIM und Mitautor des deutschen Klinik Codex/Ärzte Codex (2017/2019). 